\title{
Searching for absorbed AGN in the 2XMM-Newton pre-release EPIC Serendipitous Source Catalogue
}

\author{
E. Memola, ${ }^{1}$, A. Caccianiga ${ }^{1}$, F. Cocchia ${ }^{1}$, R. Della Ceca ${ }^{1}$, T. Maccacaro ${ }^{1}$, P. Severgnini ${ }^{1}$, \\ D. J. Fyfe ${ }^{2}$, S. Mateos ${ }^{2}$, M. G. Watson ${ }^{2}$, and G. Lamer ${ }^{3}$ \\ 1 INAF - Osservatorio Astronomico di Brera, via Brera 28, 20121 Milano, Italy \\ e-mail: elisabetta.memola@brera.inaf.it \\ 2 Department of Physics \& Astronomy, University of Leicester, Leicester LE1 7RH, UK \\ 3 AIP - Astrophysikalisches Institut Potsdam, An der Sternwarte 16, 14482 Potsdam, Germany \\ Received 14 October 2006 / Accepted 29 December 2006
}

ABSTRACT

\begin{abstract}
Aims. We aim to test a method of efficiently selecting X-ray obscured AGN in the 2XMM-Newton EPIC Serendipitous Source Catalogue.

Methods. By means of a strong correlation established using the XMM-Newton Hard Bright Sample between the intrinsic absorption and the hardness ratio to the $0.5-2.0 \mathrm{keV}$ and $2.0-4.5 \mathrm{keV}$ bands, an efficient way of selecting absorbed sources has been worked out. A hardness ratio selection based on the 2XMM-Newton pre-release Source Catalogue led us to the definition of candidates likely to be obscured in X-rays.

Results. X-ray and optical spectral analysis were performed for three objects. Strong absorption $\left(N_{\mathrm{H}}>10^{22} \mathrm{~cm}^{-2}\right)$ was detected from the X-ray analysis, confirming the efficiency of the method used to select obscured sources. The presence of absorption is also revealed in the optical band, although at a significantly lower level than inferred from the X-ray band.
\end{abstract}

Key words. surveys - methods: data analysis - X-rays: galaxies - galaxies: active

\section{Introduction}

Medium-deep X-ray surveys carried out with Chandra and XMM-Newton (see Brandt \& Hasinger 2005, and references therein for a recent review) have shown that obscured active galactic nuclei (AGN) are the main contributors to the cosmic X-ray background (XRB). Fabian \& Iwasawa (1999) found out that at least $85 \%$ of the emitted intrinsic flux of the XRB spectrum is absorbed, by assuming the absorber to be dusty gas. Therefore, understanding the characteristics of obscured AGN (i.e. luminosity and redshift distribution, spectral energy distribution, the properties of the circumnuclear medium, cosmological evolution) is a key issue in unveiling the accretion process on super massive black holes and tracing and following its evolution with cosmic time.

To efficiently select sizeable and representative samples of absorbed AGN is not an easy task, even at bright fluxes. The strong absorption that affects the intrinsic emission of these sources does not allow us to study, and in several cases even to reveal (e.g. Della Ceca et al. 2002), their nuclear properties in the optical and soft X-ray $(E<2 \mathrm{keV})$ bands. Absorbed AGN can be efficiently selected (at least the Compton-thin ones) and studied using hard X-ray $(E \sim 2-10 \mathrm{keV})$ bands where the effect of absorption is less severe and where the host galaxy contribution is minimal. Nevertheless, as shown e.g. by the XMM-Newton hard bright sample, HBS (Caccianiga et al. 2004; Della Ceca et al. 2005; Della Ceca et al. 2007, in preparation)

* Based on observations performed at the European Southern Observatory, La Silla, Chile and on observations obtained with XMM-Newton, an ESA science mission with instruments and contributions directly funded by ESA Member States and the USA (NASA). at fluxes above $\sim 7 \times 10^{-14} \mathrm{erg} \mathrm{cm}^{-2} \mathrm{~s}^{-1}$, even in the "hard" $4.5-7.5 \mathrm{keV}$ band, absorbed $\mathrm{AGN}^{1}$ represent only the $\sim 25 \%$ of the sources. It is therefore essential to increase the efficiency in selecting X-ray absorbed AGN in order to build up well-defined and sizable samples of these interesting sources.

Here we introduce a powerful method for selecting bright X-ray absorbed candidates and discuss the results of the X-ray and optical spectral analysis for three sources. Throughout this paper we adopt $H_{0}=70 \mathrm{~km} \mathrm{~s}^{-1} \mathrm{Mpc}^{-1}, \Omega_{\mathrm{M}}=0.3$, and $\Omega_{\Lambda}=$ 0.7 . We define a power-law spectrum such that $\mathrm{d} N / \mathrm{d} E=A E^{-\Gamma}$ where $N$ is the number of photons, $E$ the photon energy, $A$ the normalization, and $\Gamma$ the photon index.

\section{Bright, obscured AGN candidates in the 2XMM-Newton pre-release EPIC Serendipitous Source Catalogue}

The work presented here is based on the results from the XMM-Newton Hard Bright Sample (Caccianiga et al. 2004; Della Ceca et al. 2004; Della Ceca et al. 2005). The survey was performed in the $4.5-7.5 \mathrm{keV}$ band at a flux limit of $F_{\mathrm{x}} \sim$ $7 \times 10^{-14} \mathrm{erg} \mathrm{cm} \mathrm{cm}^{-2} \mathrm{~s}^{-1}$. The source sample is now almost fully spectroscopically-identified $(65 / 67$ sources identified), and the optical and X-ray spectral analysis is complete (Della Ceca et al. 2005; Caccianiga et al. 2007, in preparation; Della Ceca et al. 2007, in preparation). The authors found that $\mathrm{X}$-ray absorbed and unabsorbed AGN are clearly distinguishable

1 We use here and in the following $N_{\mathrm{H}}=10^{22} \mathrm{~cm}^{-2}$ as the dividing value between unabsorbed and absorbed sources in the X-rays. 
by means of the hardness ratio HR2, defined as HR2 $=\frac{C R 3-C R 2}{C R 3+C R 2}$, where $C R 2$ and $C R 3$ are the PSF and vignetting-corrected countrates in the $0.5-2.0$ and $2.0-4.5 \mathrm{keV}$ energy band, respectively (see Fig. 3 in Della Ceca et al. 2005). By considering the constraint HR2 $>0$, at the survey flux limit, they found 14 sources out of $15(\sim 93 \%)$ to have absorbing column densities higher than $\sim 10^{22} \mathrm{~cm}^{-2}$, and 4 of these sources $(\sim 30 \%)$ turned out to be the rare X-ray type 2 quasars $\left(L_{\mathrm{x}}>10^{44} \mathrm{erg} \mathrm{s}^{-1} ; N_{\mathrm{H}}>10^{22} \mathrm{~cm}^{-2}\right)$.

The XMM-Newton Survey Science Centre $(\mathrm{SSC})^{2}$ is assembling the second XMM-Newton EPIC Serendipitous Source Catalogue (Watson et al. 2007, in preparation). A first preliminary version of the 2XMM Serendipitous EPIC Source Catalogue (2XMMp) has been recently released and is available at http://xmm.vilspa.esa.es/xsa/. The catalogue contains $153105 \mathrm{X}$-ray source detections drawn from 2400 useful XMM-Newton EPIC public observations. The median flux (in the total photon energy band $0.2-12 \mathrm{keV}$ ) of the catalogue sources is $\sim 2.4 \times 10^{-14} \mathrm{erg} \mathrm{cm}^{-2} \mathrm{~s}^{-1} ; \sim 20 \%$ of the sources having fluxes below $10^{-14} \mathrm{erg} \mathrm{cm}^{-2} \mathrm{~s}^{-1}$.

The values of HR given in the catalogue are defined using energy bands that are slightly different from the ones used in the HBS. The HR closer to the HR2 used in the HBS (see above) is the HR3, defined as HR3 $=\frac{C R 4-C R 3}{C R 4+C R 3}$, where $C R 3$ and $C R 4$ are the PSF and vignetting-corrected countrates in the 1.0-2.0 and $2.0-4.5 \mathrm{keV}$ energy band, respectively. In the $2 \mathrm{XMMp}$ catalogue, there are 427 high latitude $\left(|b|>20^{\circ}\right)$ sources, which have $\mathrm{HR}^{3}>0$ and MOS2 detection likelihood (ML) in the $4.5-12 \mathrm{keV}$ energy band $>20$ (Cash 1979). This group of sources represents a crucial starting point for testing the efficiency in selecting absorbed AGN. We picked up three bright X-ray sources with $F_{\mathrm{x}}>10^{-13} \mathrm{erg} \mathrm{cm}^{-2} \mathrm{~s}^{-1}$ in the energy band $4.5-12 \mathrm{keV}$ and a bright optical counterpart $(R<17 \mathrm{mag})$ to analyse their X-ray and optical spectra. The optical counterparts were observed at the 4-m New Technology Telescope (NTT@ESO) in Chile last March 2006, as part of a more general programme concerning the Hard Bright Sample. In the following we present and discuss our results.

\section{X-ray spectral analysis}

We performed the X-ray spectral analysis of XMM-Newton data for the following sources: 2XMMpJ115121.8-283605, 2XMMpJ145442.2+182937, and 2XMMpJ151612.2+070341. They were observed between August 2000 and February 2003 with exposure times ranging from $\sim 23$ up to $\sim 42 \mathrm{ks}$ (for the observation details see Table 1). The EPIC MOS1, MOS2, and pn detectors were operated in Prime Full Window Mode.

All MOS and pn data were reprocessed with the XMM-Newton Science Analysis Software (XMM-SAS) version 6.5.0, using the latest calibration products. Source counts for MOS1, MOS2, and pn (when available) were extracted in the $0.2-12 \mathrm{keV}$ band from a circular region, centred on the source, which had a radius of 25 arcsec for 2XMMpJ115121.8-283605, 14 arcsec for 2 XMMpJ145442.2+182937, and 12 arcsec for 2XMMpJ151612.2+070341, depending on the countrate statistics and source off-axis. Background spectra were extracted

${ }^{2}$ The XMM-Newton Survey Science Centre (SSC, see http://xmmssc-www.star.le.ac.uk) is an international collaboration, involving a consortium of 10 institutions, appointed by ESA to help the SOC in developing the software analysis system, to pipeline process all the XMM-Newton data, and to exploit XMM-Newton serendipitous detections.

${ }^{3}$ HR3 refers here to MOS2 data, which were also used for the HBS. from larger source-free circular regions (radius of $\sim 40-$ 80 arcsec) close to the object. We selected single and double events (PATTERN $\leq 4)$ for the pn and single, double, triple, and quadruple events (PATTERN $\leq 12$ ) for the MOS. Our analysis is based on both EPIC MOS (MOS1 and MOS2) and pn data. In particular, for both MOS1 and MOS2, we extracted source and background spectra and then summed them up a posteriori by means of mathpha, ending up with one source spectrum and one background spectrum region valid for a global MOS1+MOS2 case. The ancillary response matrix (ARF) and the detector response matrix (RMF) were created by the XMM-SAS tasks arfgen and rmfgen. The arfgen routine convolves the mirror effective area (vignetting modified), filter transmission, and CCD efficiencies as a function of energy to return the ARF component of response. The ARF, at the source position on the detector, is emission-weighted, and the weighting falls off with distance from the source in line with the PSF. The RMF is spatially averaged using an appropriate PSF detector map. The response matrixes were also merged by using addrmf and addarf. Due to the countrate of our sources (see Table 1), pile up is not a problem.

We used the XSPEC package (version 11.3.1; Arnaud 1996) in order to perform the spectral fitting analysis. MOS and pn spectra were fitted simultaneously (when both data were available) and respectively in the 0.3-10 (2XMMpJ115121.8-283605), 0.7-10 (2XMMpJ145442.2+ 182937), and 0.5-10 (2XMMpJ151612.2+070341) keV energy range. The source counts were grouped into energy bins such that each bin contains at least 15 counts where the fit statistic in use was chi-squared $\left(\chi^{2}\right)$. The quoted errors on the best-fit parameters correspond to the $90 \%$ confidence level for one interesting parameter (i.e., $\Delta \chi^{2}=2.71$; Avni 1976). To analyse the X-ray data we made use of the redshifts derived from the optical spectra (see Sect. 4 and Table 2).

\section{1. $2 X M M p J 115121.8-283605$}

The source 2XMMpJ115121.8-283605 (see Fig. 1) was observed by XMM-Newton on January 3, 2002 for about $40 \mathrm{ks}$ of good exposure time. The pn data are not available because the source is outside the pn field of view. Fitting the data with a single absorbed power-law model leads to obvious residuals in the low-energy range. Our best-fit model $\left(\chi^{2} /\right.$ d.o.f. $\left.=27.41 / 25\right)$ is therefore based on a leaky-absorbed power-law continuum (see Table 2), where two power-laws are invoked to explain both the soft (0.3-1.0 keV - unabsorbed power-law) and the hard (up to $10 \mathrm{keV}$ - absorbed power-law) part of the X-ray spectrum. The leaky-absorbed power-law continuum has a functional form, which provides a simple and convenient parametrization of the relative contributions of both the primary absorbed component and the component that produces the flux excess at soft energies. The model might represent the physical case of an X-ray source observed both directly through the absorbing torus (absorbed power-law) and after the scattering on a warm, highly ionized gas located outside the absorbing medium (unabsorbed powerlaw). Another possible interpretation is the presence of a cloudyabsorbing medium, which would be responsible for both the absorbed and the transmitted component. Nevertheless, emission lines at soft X-rays might be present, due to nuclear processes or related to starburst activity, as well as a thermal component because of the presence of a hot plasma. We can clearly not prove this hypothesis due to the lack of soft energy statistics. The two power-laws are constrained to have the same photon index $(\Gamma \sim 1.9)$, whereas the normalization of the unabsorbed 
Table 1. XMM-Newton observation log.

\begin{tabular}{|c|c|c|c|c|c|c|c|c|}
\hline Source & Obsid & Obs.date & $\operatorname{mos} 1-\exp (\mathrm{s})$ & $\operatorname{mos} 2-\exp (\mathrm{s})$ & $\operatorname{mos} 1+\operatorname{mos} 2$-cts & pn-exp (s) & pn-cts & Filter ${ }^{*}$ \\
\hline 2XMMpJ115121.8-283605 & 0027340101 & 20020103 & 42062 & 37253 & 299 & no pn & no pn & Thin1 \\
\hline 2ХMMpJ145442.2+182937 & 0145020101 & 20030216 & 36975 & 36421 & 280 & 25535 & 274 & Thin1 \\
\hline 2ХMMpJ151612.2+070341 & 0109920101 & 20000821 & 30076 & 30123 & 84 & 23085 & 133 & Thin1 \\
\hline
\end{tabular}

* Filter type refers to all the EPIC instruments involved in the corresponding observation.

Table 2. XMM-Newton spectral analysis: best-fit parameters.

\begin{tabular}{lcccccccccc}
\hline \hline Source & $R$ & $z$ & $N_{\mathrm{H}_{\mathrm{Gal}}}^{a}$ & $N_{\mathrm{H}}^{b}$ & $\Gamma$ & $\begin{array}{c}E_{\mathrm{Fe}-\mathrm{K} \alpha} \\
\mathrm{keV}\end{array}$ & $\begin{array}{c}E W_{\mathrm{Fe}-\mathrm{K} \alpha} \\
\mathrm{eV}\end{array}$ & $\chi^{2} /$ d.o.f. & Flux-mos $^{c}$ & $L^{d}$ \\
\hline 2XMMpJ115121.8-283605 & 16.64 & 0.177 & 0.06 & $2.6_{-1.2}^{+1.4}$ & $1.9 \pm 0.5$ & - & - & $27.41 / 25$ & 1.76 & 1.8 \\
2XMMpJ145442.2+1829372 & 16.12 & 0.116 & 0.02 & $1.5_{-0.5}^{+0.8}$ & $1.4 \pm 0.3$ & $6.34_{-0.11}^{+0.44}$ & $700_{-480}^{+300}$ & $30.73 / 33$ & 2.08 & 0.7 \\
2XMMpJ151612.2+070341 & 17.20 & 0.176 & 0.03 & $14_{-4.4}^{+7.4}$ & $1.9^{*}$ & - & - & $16.55 / 12$ & 1.07 & 1.6 \\
\hline
\end{tabular}

${ }^{1}$ Model: leaky-absorbed power-law. ${ }^{2}$ Model: single-absorbed power-law + Gaussian line; ${ }^{*}$ Frozen. ${ }^{a}$ Galactic column density in units of $10^{22} \mathrm{~cm}^{-2} ;{ }^{b}$ Absorbing column density in the source direction in units of $10^{22} \mathrm{~cm}^{-2}$; ${ }^{c} \mathrm{X}$-ray fluxes in the band $2-10 \mathrm{keV}$ in units of $10^{-13} \mathrm{ergs} \mathrm{cm}^{-2} \mathrm{~s}^{-1}$ and corrected only for the Galactic absorption; ${ }^{d} \mathrm{X}$-ray luminosities in the band $2-10 \mathrm{keV}$ in units of $10^{43} \mathrm{erg} \mathrm{s}^{-1}$ and corrected for Galactic and intrinsic absorption.

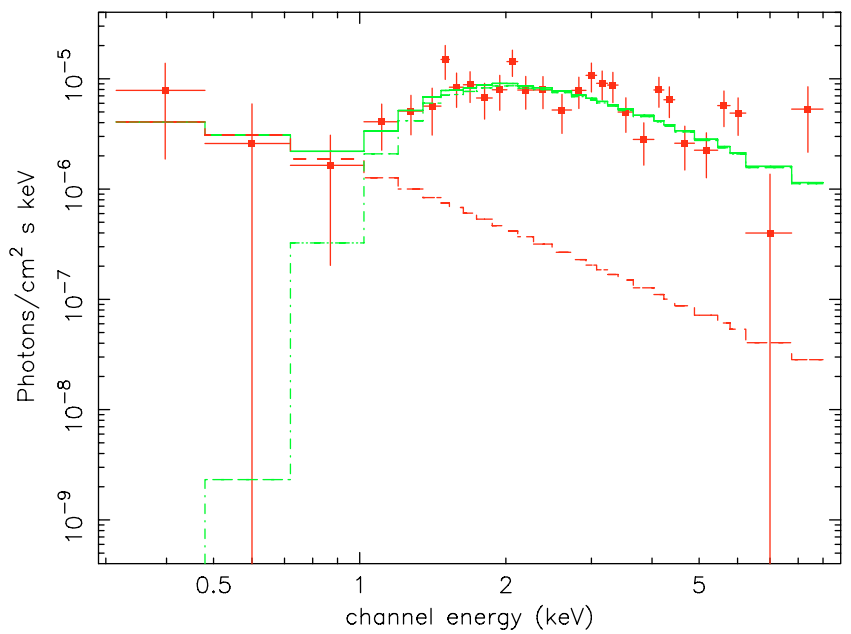

Fig. 1. 2XMMpJ115121.8-283605: XMM-Newton MOS folded spectrum. The best-fit model is a leaky-absorbed power-law.

component results in a few percent $(\sim 3 \%)$ of the normalization of the absorbed one, in agreement with previous results on absorbed AGN (e.g. Turner et al. 1997; Della Ceca et al. 2000; Caccianiga et al. 2004). The absorbed power-law reveals an intrinsic hydrogen column density, $N_{\mathrm{H}}$, of about $2.6 \times 10^{22} \mathrm{~cm}^{-2}$, which clearly points to an X-ray absorbed source (even with a single-absorbed power-law model the $N_{\mathrm{H}}$ was about $2 \times$ $\left.10^{22} \mathrm{~cm}^{-2}\right)$. The intrinsic X-ray luminosity of this source $\left(L_{\mathrm{x}} \sim\right.$ $1.8 \times 10^{43} \mathrm{erg} \mathrm{s}^{-1}$ ) is typical of a Seyfert galaxy.

\section{2. $2 X M M p J 145442.2+182937$}

The source 2XMMpJ145442.2+182937 (see Fig. 2) was observed by XMM-Newton on February 16, 2003 for about $37 \mathrm{ks}$ of good exposure time by the MOS cameras and $25 \mathrm{ks}$ by the pn camera. The best-fit model $\left(\chi^{2} /\right.$ d.o.f. $\left.=30.73 / 33\right)$ based on both MOS and pn data is a single absorbed power-law along with a Gaussian line detection at $90 \%$ confidence level (see Table 2). The power-law shows a photon index $\Gamma \sim 1.4$, together with $N_{\mathrm{H}} \sim 1.5 \times 10^{22} \mathrm{~cm}^{-2}$, which points to an X-ray absorbed source. If we freeze the photon index to $\Gamma \sim 1.9$, the $N_{\mathrm{H}}$ value remains

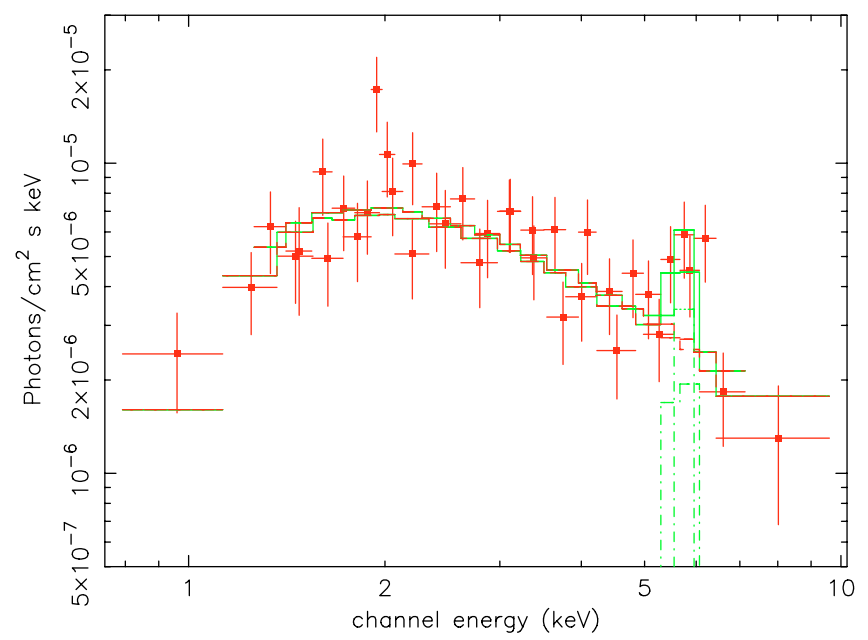

Fig. 2. 2XMMpJ145442.2+182937: XMM-Newton MOS+pn folded spectrum. The best-fit model is a single-absorbed power-law with a Gaussian line.

quite stable around $\sim 2.4 \pm 0.4 \times 10^{22} \mathrm{~cm}^{-2}$. In both cases the iron line equivalent width is about $700 \mathrm{eV}$ with associate errors of about $60 \%$. This equivalent width value, due to the large error associated to it, is consistent with the value of $N_{\mathrm{H}}$ measured from the cut-off. Moreover, a detailed analysis of the line properties is not warranted by the quality of the data, therefore we also can not exclude a possible blending of two narrow iron lines at 6.4 and $6.7 \mathrm{keV}$. The X-ray luminosity $\left(L_{\mathrm{x}} \sim 0.7 \times 10^{43} \mathrm{erg} \mathrm{s}^{-1}\right)$ is typical of a Seyfert galaxy.

\section{3. $2 X M M p J 151612.2+070341$}

The source 2XMMpJ151612.2+070341 (see Fig. 3) was observed by XMM-Newton on August 21, 2000 for about $30 \mathrm{ks}$ of good exposure time with the MOS cameras and $23 \mathrm{ks}$ with the pn camera. A single-absorbed power-law model $\left(\chi^{2} /\right.$ d.o.f. $=$ $18.98 / 12$ ) provided a best-fit value of the photon index that was very low $(\Gamma \sim 0.5)$, whereas the value of $N_{\mathrm{H}}$ was already high and about $\sim 4 \times 10^{22} \mathrm{~cm}^{-2}$. Such a flat $\Gamma$ value would suggest Compton thickness, a thesis otherwise not supported by the 


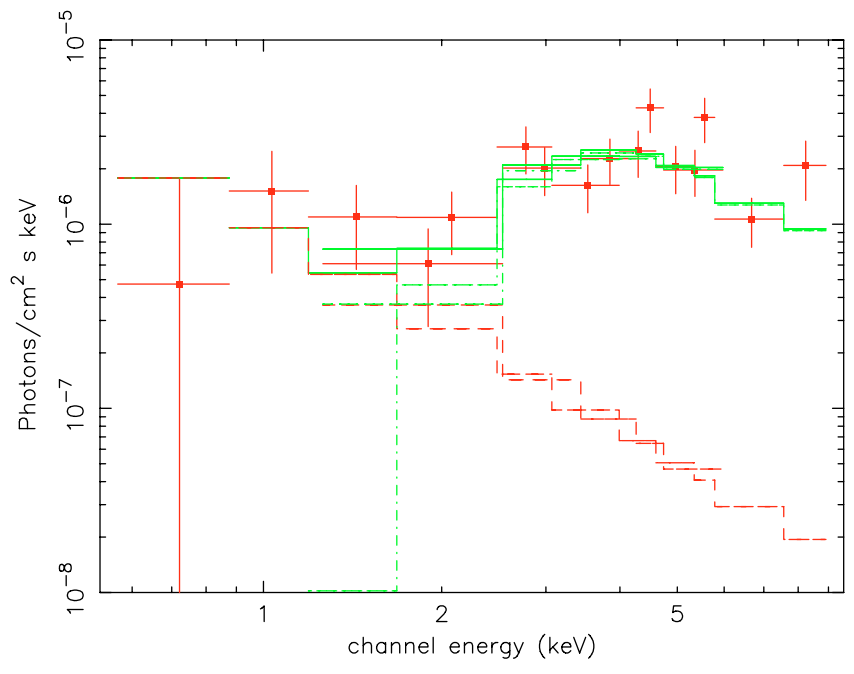

Fig. 3. 2XMMpJ151612.2+070341: XMM-Newton MOS+pn folded spectrum. The best-fit model is a leaky-absorbed power-law.

optical data. After freezing the value of $\Gamma$ to 1.9 , more typical of AGN, there were residuals at $1-2 \mathrm{keV}$. The best-fit model $\left(\chi^{2} /\right.$ d.o.f. $\left.=16.55 / 12\right)$ is a leaky-absorbed power-law continuum with a frozen photon index of 1.9 (see Table 2). The statistics do not allow us to leave this parameter free. The hydrogen column density is $N_{\mathrm{H}} \sim 1.4 \times 10^{23} \mathrm{~cm}^{-2}$, which points to an X-ray absorbed object. The X-ray luminosity $\left(L_{\mathrm{x}} \sim 1.6 \times\right.$ $10^{43} \mathrm{erg} \mathrm{s}^{-1}$ ) is typical of a Seyfert galaxy.

\section{Optical spectral analysis}

We performed the spectral analysis of the optical counterparts for the three X-ray sources. Optical images and spectroscopy were acquired at the NTT@ESO telescope using EMMI. We obtained shallow optical images with a typical exposure time of 60-90 s. Optical images were bias-subtracted, flat-field divided, and flux-calibrated using observations of standard stars obtained during each night. Astrometric calibration was performed using the software package GAIA (version 2.6-9 by P.W. Draper) by matching sources found in the USNO catalogue (Monet et al. 1998). We show the $R$-images of our sources in Figs. 4-6 (left panels) and report their $R$-magnitudes in Table 2.

Long-slit spectroscopy was carried out in the 3800-9000 $\AA$ band with a low dispersion $(\sim 1.7 \AA /$ pixel), long-slit configuration and with a low-resolution grism (Grism\#2, $R \sim 600$ ). The seeing during the observing run ranged from $1^{\prime \prime}$ to $2^{\prime \prime}$, and we used a slit width of $1^{\prime \prime}-1.5^{\prime \prime}$. Data reduction was performed using IRAF ${ }^{4}$ standard packages. Wavelength calibrations were carried out by comparison with exposures of He-Ar lamps. The relative flux calibration of the spectra was obtained using observations of spectro-photometric standard stars performed within a few hours of the spectroscopy of our sources; no attempt was made to obtain an absolute flux calibration of the spectra.

The optical spectra of the three objects are presented in Figs. 4-6, (right panels). The spectra show relatively strong emission lines, like the [OIII] $\lambda 5007 \AA$ and the $\mathrm{H} \alpha$-[NII] blend, and a continuum that is highly contaminated (or dominated) by the light of the host galaxy. The presence of broad

${ }^{4}$ IRAF is distributed by the National Optical Astronomy Observatory, that is operated by the Association of Universities for Research in Astronomy, Inc., under cooperative agreement with the National Science Foundation.
$\left(F W H M \sim 1900-3000 \mathrm{~km} \mathrm{~s}^{-1}\right)$ emission lines is clear in one source (2XMMpJ151612.2+070341) and is also suggested in 2XMMpJ115121.8-283605, where the $\mathrm{H} \alpha$ is likely to have a broad component. In 2XMMpJ145442.2+182937, instead, only narrow lines are visible in the spectrum.

To estimate the level of optical absorption in the three sources, we made use of the method described in Caccianiga et al. (2007, in preparation). They apply this procedure to studying and classifying the objects in the XMM-Newton Bright Serendipitous Survey that are contaminated by the host galaxy light ("diluted" sources). In synthesis, this approach involves a spectral model composed of a galaxy plus an AGN template (Severgnini et al. 2003). The AGN template, in particular, has a component (the continuum and the broad emission lines) that can be absorbed according to an appropriate extinction curve and a second component (the narrow emission lines) that is not affected by the absorption. We estimate the optical absorption as the value that best emulates the observed spectrum (both the continuum and the emission-line intensity), paying particular attention to correctly reproducing the strength of some critical lines, like the [OIII] $\lambda 5007 \AA$ and the $\mathrm{H} \alpha$. We find that two sources (2XMMpJ145442.2+182937 and 2XMMpJ115121.8-283605) are affected by a relatively high absorption $\left(A_{\mathrm{V}}>2\right.$ mag and $A_{\mathrm{V}} \sim 2 \mathrm{mag}$ ), while the third (2XMMpJ151612.2+070341) is only moderately absorbed ( $A_{\mathrm{V}} \sim 1$ mag). When translated into a value of $N_{\mathrm{H}}$ using the standard Galactic relation (e.g. Maiolino et al. 2001a), the absorption levels inferred from the optical spectra are $N_{\mathrm{H}} \geq(2-4) \times 10^{21} \mathrm{~cm}^{-2}$, i.e. lower (significantly lower, in the case of $2 \mathrm{XMMpJ} 151612.2+070341)$ than the values obtained from the X-ray data (see Sect. 3).

The existence of a fraction of AGN where the level of absorption deduced by the X-ray spectra is significantly higher than what can be inferred from the optical data is well known and discussed in the literature (e.g. Maccacaro et al. 1982; Maiolino et al. 2001b; Cappi et al. 2006). To date, we are not in a position of quantifying the frequency of this kind of objects since the sources discussed here do not constitute a complete, well-defined sample of objects. It is very likely that the way we have chosen these sources out of the 2XMMp catalogue, in particular the requirement of a bright optical counterpart, has favoured the selection of objects that are highly absorbed in the $\mathrm{X}$-rays, but with a low/moderate optical absorption. A larger and unbiased sample of HR-selected sources would be instrumental in evaluating the fraction of sources strongly absorbed in the $\mathrm{X}$-rays but only moderately absorbed in the optical band.

\section{Concluding remarks}

Three sources of the 2XMMp catalogue with hard X-ray colour strongly suggesting the presence of absorption were also observed at NTT@ESO. All three sources show high absorption $\left(N_{\mathrm{H}}>10^{22} \mathrm{~cm}^{-2}\right)$ in the X-ray band and moderate absorption ( $\left.A_{\mathrm{V}} \sim 1-2 \mathrm{mag}\right)$ in the optical. We can therefore definitely state that we are dealing with a successful selection criterion able to "isolate" obscured sources in X-rays. Page et al. (2007) have been studying hard-spectrum XMM-Newton sources with $2-4.5 \mathrm{keV} X$-ray flux $>10^{-14} \mathrm{erg} \mathrm{cm}^{-2} \mathrm{~s}^{-1}$ and get the same high efficiency in finding absorbed X-ray sources $\left(6.3 \times 10^{21}<N_{\mathrm{H}}<\right.$ $2.5 \times 10^{23} \mathrm{~cm}^{-2}$ ).

We note that, of the large number (427) of high-latitude sources with HR3 $>0$ and ML $>20$ available in the $2 \mathrm{XMM}$ pre-release Serendipitous EPIC Source Catalogue, 381 have a MOS2 X-ray flux in the $4.5-12 \mathrm{keV}$ band larger than $10^{-13} \mathrm{erg} \mathrm{cm}^{-2} \mathrm{~s}^{-1}$. This means that their optical spectroscopy 

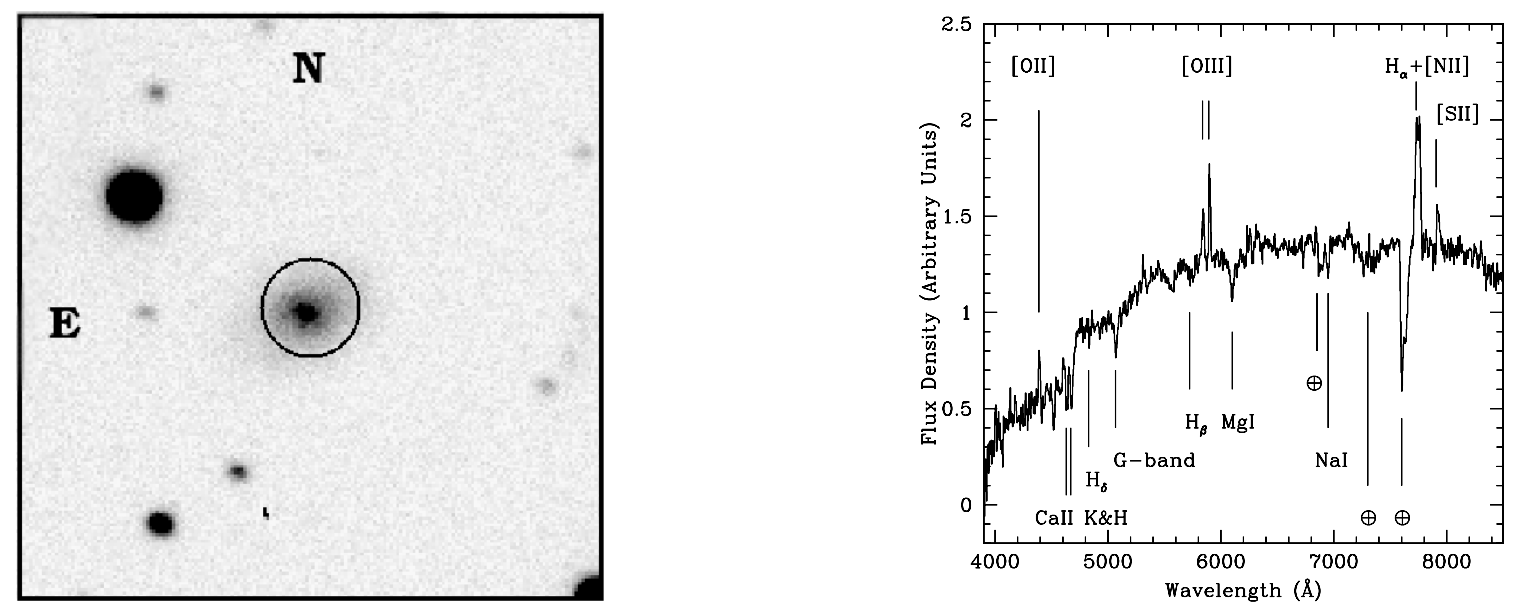

Fig. 4. 2XMMpJ115121.8-283605: NTT@ESO $R$-image $\left(1^{\prime} \times 1^{\prime}\right)$. North is on top and east to the left. A circle of $5^{\prime \prime}$ radius, corresponding to the $95 \%$ confidence level X-ray error circle (see Della Ceca et al. 2004), is shown (left panel) to clearly mark the optical counterpart of the XMM-Newton source; NTT@ESO optical spectrum (right panel).
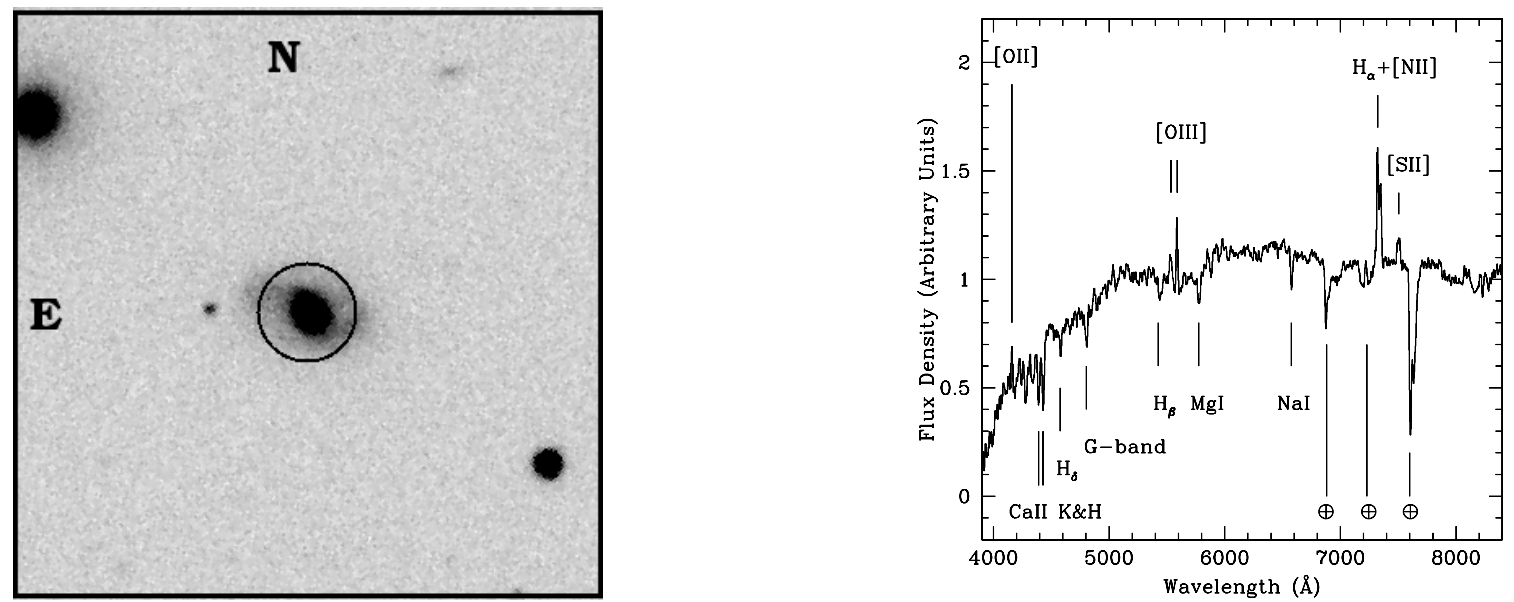

Fig. 5. $2 X M M p J 145442.2+182937$ : NTT@ESO $R$-image $\left(1^{\prime} \times 1^{\prime}\right)$. North is on top and east to the left. A circle of $5^{\prime \prime}$ radius, corresponding to the $95 \%$ confidence level X-ray error circle (see Della Ceca et al. 2004), is shown (left panel) to clearly mark the optical counterpart of the XMM-Newton source; NTT@ESO optical spectrum (right panel).
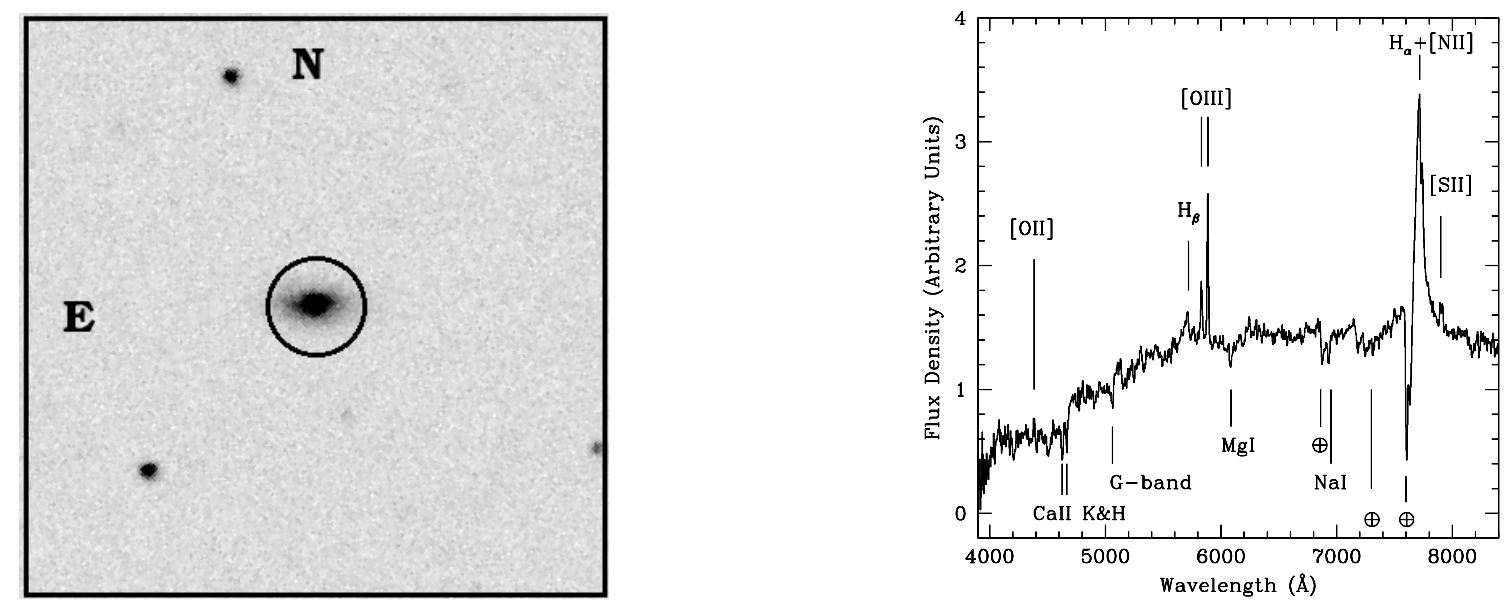

Fig. 6. $2 \mathrm{XMMpJ} 151612.2+070341$ : NTT@ESO $R$-image $\left(1^{\prime} \times 1^{\prime}\right)$. North is on top and east to the left. A circle of $5^{\prime \prime}$ radius, corresponding to the $95 \%$ confidence level X-ray error circle (see Della Ceca et al. 2004), is shown (left panel) to clearly mark the optical counterpart of the XMM-Newton source; NTT@ESO optical spectrum (right panel). 
should be achievable with ground-based telescopes, as already happened for most of the sources of the HBS. We can therefore foresee a valuable increment to the known sample of absorbed AGN, up to a few hundred newly absorbed AGN, and about $1 / 3$ among them, as inferred from the HBS statistics, should be the rare type 2 quasars.

Acknowledgements. We acknowledge financial contribution from the contract ASI-INAF I/023/05/0. Part of this work was supported by a Cofin grant for the year 2006

\section{References}

Arnaud, K. A. 1996, ASPC, 101, 17

Avni, Y. 1976, 210, 642

Brandt, W. N., \& Hasinger, G. 2005, ARA\&A, 43, 827
Caccianiga, A., Severgnini, P., Braito, V., et al. 2004, A\&A, 416, 901 Cappi, M., Panessa, F., Bassani, L., et al. 2006, A\&A, 446, 459 Cash, W. 1979, ApJ, 228, 939

Della Ceca, R., Maccacaro, T., Rosati, P., et al. 2000, A\&A, 355, 121 Della Ceca, R., Ballo, L., Tavecchio, F., et al. 2002, ApJ, 581, L9 Della Ceca, R., Maccacaro, T., Caccianiga, A., et al. 2004, A\&A, 428, 383 Della Ceca, R., Caccianiga, A., Severgnini, P., et al. 2005, Proc. of The X-ray Universe 2005, San Lorenzo de El Escorial (Spain) organized by ESAESAC [arXiv: astro-ph/0510845]

Fabian, A. C., \& Iwasawa K. 1999, MNRAS, 303, L34

Maccacaro, T., Perola, G.C., \& Elvis, M. 1982, ApJ, 257, 47

Maiolino, R., Marconi, A., Salvati, M., et al. 2001a, A\&A, 365, 28

Maiolino, R., Marconi, A., \& Oliva, E. 2001b, A\&A, 365, 37

Monet D. Bird A., Canzian, B., Dahn, C., et al. 1998, VizieR Online Data Catalog, 1252

Page, M. J., Lehmann, I., Boller, Th., et al. 2007, MNRAS, submitted Severgnini, P., Caccianiga, A., Braito, V., et al. 2003, A\&A, 406, 483

Turner, T. J., George, I. M., Nandra, K., et al. 1997, ApJ, 488, 164 